\title{
Microfabricated emitter array for an ionic liquid electrospray thruster
}

\author{
Kaito Nakagawa $^{1^{*}}$, Toshiyuki Tsuchiya ${ }^{2}$, and Yoshinori Takao ${ }^{3 *}$ \\ ${ }^{1}$ Department of Systems Integration, Yokohama National University, Yokohama 240-8501, \\ Japan \\ ${ }_{2}^{2}$ Department of Micro Engineering, Graduate School of Engineering, Kyoto University, Kyoto \\ 615-8540, Japan \\ ${ }^{3}$ Division of Systems Research, Yokohama National University, Yokohama 240-8501, Japan \\ *E-mail: nakagawa-kaito-mf@ynu.jp; takao-yoshinori-yk@ynu.jp
}

\begin{abstract}
We have fabricated needle-shaped emitters on a Si wafer by a MEMS process, and measured the voltage-current characteristics and the frequency dependence of a bipolar pulse voltage for ionic liquid electrospray thrusters, which can be mounted on nanosatellites $(\leq \sim 10 \mathrm{~kg})$. Although the extracted current did not increase with increasing number of emitters, probably owing to the lack of uniformity of the emitters fabricated, we have demonstrated that the emitted current depends on the gap distance between the emitter and the extractor grid electrode, and low frequencies of the bipolar pulse voltage are desirable for thruster operation. Moreover, the Bosch process is required for fabricating a reservoir of ionic liquid, which prevents undesirable
\end{abstract}


electrical short circuits, and the minimum emitter pitch to prevent loss of ion beams to the extractor is estimated to be about $400 \mu \mathrm{m}$. 


\section{Introduction}

The electrospray technique is applied in various fields, such as focused ion beam (FIB), ${ }^{1)}$ deposition/etching/sputtering, ${ }^{2,3)}$ mass spectrometry, ${ }^{4,5)}$ and field emission electric propulsion (FEEP). ${ }^{6}$ Electrospray thrusters presented here are one of the electric propulsion systems using electrostatic acceleration.

Electric propulsion is one of the space propulsion systems. To obtain thrust, this propulsion exhausts high-velocity ions or plasmas, which are accelerated by electrical heating and/or electric and magnetic body forces, and is normally categorized as electrothermal, ${ }^{7-9)}$ electrostatic, ${ }^{10,11)}$ or electromagnetic propulsion. ${ }^{12,13)}$ Compared with chemical propulsion producing a large thrust, electric propulsion generates a very small thrust, but its specific impulse is very large (i.e., high fuel efficiency). As the specific impulse of thrusters becomes larger, spacecraft can operate for a longer time with less amount of propellant. For electrostatic propulsion, the thrust $T$ and the specific impulse $I_{\mathrm{sp}}$ can be respectively defined as

$$
\begin{gathered}
T=I_{\mathrm{b}} \sqrt{2 V_{\mathrm{b}} \frac{m}{q}} \\
I_{\mathrm{sp}}=\frac{T}{\dot{m} g_{0}}=\frac{1}{g_{0}} \sqrt{2 V_{\mathrm{b}} \frac{q}{m}},
\end{gathered}
$$

where $I_{\mathrm{b}}$ is the ion beam current, which is related to the ion mass flow rate, $V_{\mathrm{b}}$ is the voltage for ion acceleration, $m$ is the ion mass, $q$ is the charge, $\dot{m}$ is the mass flow rate, and $g_{0}$ is the gravitational acceleration. ${ }^{14)}$ From Eqs. (1) and (2), it can be found that the mass-to-charge ratio is the important factor, which is dependent on the kind of propellant used. Xenon is usually used as the propellant for ion and Hall thrusters because its mass-to-charge ratio is large and it 
is not hazardous to handle. ${ }^{14)}$

Electric propulsion systems have been mounted on many spacecraft for various missions since the 1960s, such as communication, weather forecast, and deep space exploration. ${ }^{15,16)}$ Since these spacecraft are very large and complicated, they take a long time and are costly to develop. For this reason, miniature satellites (less than $100 \mathrm{~kg}$ ) have recently been attracting much interest from the space engineering community for various missions. ${ }^{17-21)}$ Since miniature satellites could be less costly and developed in a shorter term, they can be manufactured and operated by small organizations, such as universities and small companies. However, because of the limitations of weight, volume, and power, almost all of the miniature satellites do not have any propulsion systems. Without propulsion systems, the miniature satellites cannot change their orbit by themselves, which means that missions using miniature satellites are limited. That is why the development of high-performance microthrusters is required. ${ }^{15,22)}$ Ion thrusters are one of the candidate microthrusters and were already installed on the 50-kg-class microspacecraft HODOYOSHI-4 and successfully operated in space. ${ }^{21)}$ However, it is difficult to miniaturize ion thrusters further for satellites lighter than about $10 \mathrm{~kg}$ (nanosatellites) as long as the gas propellant (e.g., xenon) is employed, which requires a bulky and heavy gas feed system.

Ionic liquid electrospray thrusters (ILESTs) have recently been recognized as promising microthrusters because they do not require a gas feed system or a neutralizer. That is, the entire propellant system could be smaller and mounted on nanosatellites. Ionic liquids 
are composed of only positive and negative ions (cations and anions), and the vapor pressure is almost zero owing to the strong Coulomb force between the cations and the anions. ${ }^{23)}$ Hence, ionic liquids can exist as a liquid phase in vacuum for a long time. Because of this unique characteristic, ionic liquids have also attracted attention as promising materials, ${ }^{24,25)}$ which have been applied to, for example, lithium-ion batteries, ${ }^{26)}$ polymer actuators, ${ }^{27)}$ and solar cells. ${ }^{28)}$ Regarding the merits of using ionic liquids as propellants, they are less hazardous and easier to handle than liquid metals such as cesium used in FEEPs.

In this study, some experiments on emitter fabrication and current measurements were conducted for the development of ILESTs. Here, we report a fabrication method for an emitter array by a MEMS process, the voltage-current characteristics of ion beams, and the frequency dependence of bipolar pulse voltage. Compared with the voltage-current characteristics, the dependence on frequency in a wide range has not been reported so far. In this study, experiments were conducted in the frequency range of DC-50 Hz. In Sect. 2, the electrospray principle is described, followed by a preliminary experiment of voltage-current characteristics using a fabricated four-emitter chip. More than one hundred emitters were also fabricated and some of the highlight data obtained from the experiments are presented. Finally, a summary is presented in Sect. 5.

\section{Electrospray principle}

A schematic of an ILEST is shown in Fig. 1. The ILEST is mainly composed of emitters, an 
extractor grid, and a reservoir to stock an ionic liquid. Owing to the high voltage of about a few $\mathrm{kV}$ between the emitters and the extractor, the ionic liquid flows from the reservoir to the tip of emitters. Because the curvature radius of the emitter tips is very small ( $\sim$ a few $\mu \mathrm{m})$, a strong electric field can be generated at the emitter tip. By this strong field, the Taylor cone of the ionic liquid is formed at the tip. Here, the Taylor cone has a constant half angle $\left(49.3^{\circ}\right)$ regardless of the kind of ionic liquid used, the applied voltage, or the shape of tips. ${ }^{29)}$ When the electric field is stronger than the surface tension of the ionic liquid, ions are extracted from the ionic liquid. This effect is referred to as field emission. Subsequently, ions can be accelerated and high velocity/momentum is obtained from the potential difference between the emitters and the extractor. By exhausting these ions with a large momentum, the ILEST can obtain a thrust with a large specific impulse.

It is necessary to maintain the electrical neutrality of the entire spacecraft because there is no ground in space. In order to continuously operate electrostatic thrusters, which normally exhaust only positive ions, electrons must also be emitted from electron sources (neutralizers). ${ }^{14)}$ In contrast to conventional electrostatic thrusters, the ILEST can also exhaust negative ions by applying the opposite potential difference. Hence, neutralizers are not required for the ILEST if the thruster is operated in the following two ways. One is applying positive and negative voltages alternately, ${ }^{30)}$ and the other is simultaneously operating two thruster units spraying in opposite polarities. ${ }^{31)}$ Moreover, the molecular weight of negative ions for typical ionic liquids is similar to that of positive ions. For example, 1-ethyl-3-methylimidazolium 
tetrafluoroborate $\left(\mathrm{EMI}-\mathrm{BF}_{4}\right)$ consists of $\mathrm{EMI}^{+}$with a molecular weight of 100.08 and $\mathrm{BF}_{4}{ }^{-}$with a molecular weight of 86.80, and its structural formula is shown in Fig. 2. Although neutralizers cannot obtain a thrust owing to the very low mass of electrons, the ILEST can obtain a thrust by emitting negative ions.

The starting voltage of field emission, $V_{\text {start, }}$ is obtained from the following equation:

$$
V_{\text {start }}=\sqrt{\frac{\gamma R_{\mathrm{c}}}{\varepsilon_{0}}} \ln \left(\frac{4 d}{R_{\mathrm{c}}}\right),
$$

where $\gamma$ is the surface tension of the ionic liquid, $R_{\mathrm{c}}$ is the curvature radius of the emitter tip, $\varepsilon_{0}$ is the dielectric constant of vacuum, and $d$ is the distance between the emitters and the extractor. ${ }^{32)}$ When the distance is typically about $50-500 \mu \mathrm{m},{ }^{33,34)}$ it is found that the curvature

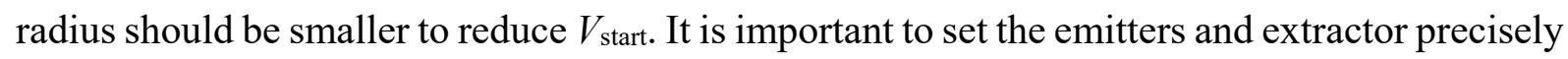
at a short distance from each other and to fabricate emitters with sharp tips.

\section{Preliminary experiment}

First, we fabricated only a few emitters on a Si wafer to establish the method of fabricating emitters and estimate the ion current that is equivalent to the thrust using Eq. (1).

\subsection{Fabricating a trial emitter chip}

We fabricated a trial emitter chip, which has an array of emitters on a silicon wafer, as shown in Fig. 3. Firstly, a positive-type photoresist (PMER P-LA900PM) was deposited on the silicon wafer and heated. This positive-type photoresist was transcribed from a photomask pattern by 
photolithography. We performed isotropic etching using $\mathrm{SF}_{6}$ to form the cone shape of the emitter tips based on the patterned positive photoresist layer. The reservoir of the ionic liquid ( $8 \mathrm{~mm}$ in diameter) was then formed by the Bosch process and the photoresist was removed. Figure 4 shows a photograph of the emitter chip and scanning electron microscopy (SEM) images of the emitters fabricated and one of their tips. The radius and height of the emitters are 75 and $170 \mu \mathrm{m}(100 \mu \mathrm{m}$ depth for the Bosch process $)$, respectively, and the pitch of the emitters is $500 \mu \mathrm{m}$. Figure $4(\mathrm{c})$ shows that the radius of the tips is smaller than $5 \mu \mathrm{m}$. Here, the wafer was singulated into a $1 \times 1 \mathrm{~cm}^{2}$ emitter chip by mechanical dicing, as shown in Fig. 4(a).

\subsection{Experimental equipment}

Figure 5(a) shows a schematic of the experimental setup for the current measurement of both the cation and anion beams and the configuration of the emitter chip, extractor grid, and collector electrode. Most experimental systems (emitters, extractor, and collector) are in a vacuum chamber evacuated by a rotary pump and a turbomolecular pump at a base pressure lower than $2.0 \times 10^{-3} \mathrm{~Pa}$. Here, the distance between the emitter tip and extractor grid is set at $0.5 \mathrm{~mm}$, the number of emitters is four, the ionic liquid employed is $\mathrm{EMI}_{-\mathrm{BF}}$, and the extractor grid is a TEM grid made of copper with a thickness of $25 \mu \mathrm{m}$ and a diameter of $3.0 \mathrm{~mm}$ [Fig. $5(b)]$.

Voltage alternation was employed to prevent the electrochemical decomposition of the ionic liquid and to neutralize the space charge, ${ }^{30)}$ where a function generator (Matsusada eK- 
FGJ) generated a bipolar pulse signal that was amplified with a high voltage amplifier (Matsusada HJOPS-4B10). The beam current of ions passing through the extractor grid was measured with a shunt resistor of $1 \mathrm{k} \Omega$ connected to the collector in series using a digital multimeter (Keithley 2110). The current intercepted by the extractor grid was also measured similarly.

\subsection{Results and discussion}

Figure 6 shows the measured ion beam currents vs applied voltage, where the repetition frequency of the bipolar pulse was set at $0.5 \mathrm{~Hz}$. When the absolute applied voltage was more than $3200 \mathrm{~V}$, ion beam currents were detected at the extractor and collector. Since only the collector current becomes the thrust force, the thrust with four emitters at $3700 \mathrm{~V}$ is estimated to be $0.77 \mu \mathrm{N}$ using Eq. (1) with the assumption that only monomers of EMI ${ }^{+}$are ejected. This value is much smaller than the thrust required for a small propulsion system mounted on nanosatellites. Note that ion beams are composed of not only monomers but also dimers, trimers, and even droplets under certain conditions. ${ }^{34,35)}$ If heavier particles were included, the thrust would increase. On the other hand, ion beams have a divergence angle, which results in the loss of directional velocity and thus a decrease in thrust. ${ }^{14)}$ Direct thrust measurement is required to obtain the actual thruster performance.

Figure 6 also indicates that there is almost no significant difference between the collector and extractor currents. To increase the thrust of the ILEST, almost all the extracted 
ions must be collected with the collector without the collisions with the extractor grid, representing the thrust loss. It can be found that the half of the thrust is lost owing to the collisions. One of the reasons was probably due to the misalignment between the central axes of the emitter and the extractor grid, which was aligned visually and manually in this experiment.

For current measurements of the collector and the extractor grid, the current values were very unstable and electrical short circuits frequently occurred between the emitter and the extractor; thus, we were unable to reduce the gap distance between the emitter and the extractor to smaller than $0.5 \mathrm{~mm}$. For each measurement, a small amount of EMI-BF4 was deposited on the emitters and reservoir. Before the deposition, the fabricated chip was exposed to atmospheric-pressure helium plasma discharges: this exposure improved the wettability of the ionic liquid on the Si surface. However, despite the wettability improvement, it was difficult to spread the ionic liquid over the reservoir uniformly except for the emitter region. The nonuniform distribution of the ionic liquid resulted in the situation that at some areas of the reservoir, the distance between the surface of the ionic liquid and the extractor grid was comparable to that between the emitter tip and the extractor grid, which seemed to frequently cause the electrical short circuit between the ionic liquid and the extractor grid. In this sense, as many emitters as possible should be fabricated in the reservoir area.

\section{Main experiment}

Results of the preliminary experiment showed that it is necessary to increase the number of 
emitters and to improve the positional accuracy of the emitters and extractor grid to increase the thrust of the ILEST.

\subsection{Fabrication of a dense emitter array}

A dense emitter array was fabricated on the surface of a silicon wafer as described in Sect. 3. To increase thrust density, the uniformity of emitters is important. As shown in the preliminary experiment, we fabricated as many emitters as possible within a circle of $3 \mathrm{~mm}$ diameter, which was the same as the diameter of the transmission electron microscopy (TEM) grid employed. We found that the rate of isotropic etching using $\mathrm{SF}_{6}$ was different between the emitters on the outermost area of the 3-mm-diameter circle and those on the central area of the circle. This result would probably be due to the difference in emitter density depending on the position of emitters, since we fabricated the emitters in the 8-mm-diameter reservoir. To improve uniformity, we placed as many emitters as possible with 250 and $500 \mu \mathrm{m}$ pitches inside the reservoir of $8 \mathrm{~mm}$ diameter. When we conducted isotropic etching, however, its rate was still different between the outermost and the central areas; the etch rate in the outermost area was higher than that in the center area, and part of the thick photoresist was lost during isotropic etching.

Figure 7 shows the mask layout for improving the uniformity of both 250- and 500$\mu \mathrm{m}$ pitch emitter chips. Since the rate of isotropic etching differed depending on the reservoir area, the emitter diameter increased with the increase in the radial distance of the emitter 
position from the center of the reservoir. We set the emitter diameter in the outermost area to be $120 \mu \mathrm{m}$, that between the outermost and the center area to be $110 \mu \mathrm{m}$, and that in the center area to be $100 \mu \mathrm{m}$. Figure 8 shows typical examples of the SEM images of the emitter array with a $250 \mu \mathrm{m}$ pitch. Although the diameters of emitter tips were still somewhat different depending on the area, the differences were reduced and almost all of the emitters existed after the Bosch process without the loss of the thick photoresist. Here, the height of the emitters was $230 \mu \mathrm{m}\left(160 \mu \mathrm{m}\right.$ depth for the Bosch process), and the wafer was singulated into a $1 \times 3 \mathrm{~cm}^{2}$ emitter chip by mechanical dicing. Figure 9 shows a photograph of an emitter chip and an extractor grid electrode. The extractor grid was made of stainless steel with a thickness of 80 $\mu \mathrm{m}$ (each grid diameter was set to be 200 and $300 \mu \mathrm{m}$ for the 250 - and $500-\mu \mathrm{m}$-pitch emitter chips, respectively) and was fabricated by metal etching.

\subsection{Experimental equipment}

The experimental equipment in the main experiment was almost the same as that in the preliminary experiment. Only the alignment system for the emitter chip and the extractor grid was improved to align the axis of the emitters and apertures of the extractor grid precisely by fine-tuning the position of both the emitter and the extractor. Figure 10 shows a cross-sectional view and the wiring diagram for the current measurement. The sides in the longitudinal direction were used for the electrical connections and the mechanical support of each electrode to the alignment system. 


\subsection{Results and discussion}

4.3.1 Voltage-current characteristics. Figure 11 shows the extractor and collector currents measured by applying a bipolar pulse voltage between the emitter chip and the extractor grid with gap distances of $d=0.2,0.3$, and $0.4 \mathrm{~mm}$ for the $500-\mu \mathrm{m}$-pitch emitter chip. The repetition frequency was also set at $0.5 \mathrm{~Hz}$. Compared with Fig. 6 in the preliminary experiment, we were able to reduce the gap distance to $0.2 \mathrm{~mm}$ and significantly suppress extractor currents owing to the improvement of the alignment of the emitter chip and extractor grid and the more uniform distribution of the ionic liquid in the dense emitter array. However, the maximum current did not increase as expected with the increase in the number of emitters. This would probably be due to the nonuniformity of the emitter tips, as shown in Fig. 8. Ions were considered to be extracted from only a fraction of many emitters, indicating that further improvement of emitter uniformity is required for better ion extraction.

As shown in Fig. 11, starting voltages of ion extraction increased with increasing gap distance; the absolute starting voltages were measured to be about 1600,2100 , and $2600 \mathrm{~V}$ for $d=0.2,0.3$, and $0.4 \mathrm{~mm}$, respectively, for both cations and anions. Although the starting voltage was almost the same for cations and anions, the extraction tendency differed. More currents were obtained for cations than for anions at all the gap distances, probably because anion beams contain more dimers $\left(\mathrm{EMI}_{-} \mathrm{BF}_{4}\right) \mathrm{BF}_{4}{ }^{-}$or droplets than cations, although time-of-flight (ToF) measurements are required for identifying the charged species, which is left for future work. The collector current increased with increasing gap distance for both cations and anions because 
of the higher potential drop between the emitter and the extractor grid, while the extractor currents also increased, which resulted in the loss of the thrust. The high voltage increases the electric field at the emitter chip, resulting in the higher initial energy of ion beams at the emitter tip. This effect would also contribute to the higher speed of ion beams at larger gap distances. Note that at a gap distance of $0.5 \mathrm{~mm}$, the extractor current increased to be comparable to the collector current; thus, we do not discuss this result hereafter. This current increase may be due to the divergence angle of the beams and smaller diameter of each extractor grid $(300 \mu \mathrm{m})$ than of the TEM grid $(450 \mu \mathrm{m})$ employed in the preliminary experiment; a slight misalignment of the emitter and extractor would result in a large current at the extractor grid.

Since the maximum amplitude $V_{\text {ap }}$ of the high-voltage amplifier is $4 \mathrm{kV}$, we applied a higher voltage than that shown in Fig. 11. However, stable currents were not obtained in this higher voltage region. Figure 12 shows a typical example of the applied voltage, collector current, and extractor current as functions of time at $V_{\text {ap }}=2200$ and $3000 \mathrm{~V}$. Both the collector and the extractor currents were stable at $V_{\text {ap }}=2200 \mathrm{~V}$ and the collector current was larger than the extractor current. However, when the applied voltage was increased further, e.g., at $V_{\text {ap }}=$ $3000 \mathrm{~V}$, both the collector and extractor currents were unstable and the extractor current was almost the same or sometimes higher than the collector current. Since the applied voltage also sometimes dropped below the set value of $3000 \mathrm{~V}$ (e.g., see at $410.3 \mathrm{~s}$ ), an electrical short circuit occurred between the emitter and the extractor grid through the ionic liquid. A previous study showed that the ion source became unstable and more than one emission site from a single 
emitter tip was observed at higher applied voltages. ${ }^{36)}$ The stable range of applied voltages was measured to be about $800 \mathrm{~V}$ from the starting voltage for all the gap distances examined in this study.

Figure 13 shows the extractor and collector currents as functions of applied voltage with a gap distance of $0.2 \mathrm{~mm}$ for the $250-\mu \mathrm{m}$-pitch emitter chip. The starting voltage shows almost the same tendency as that shown in Fig. 11(a). However, the extractor current was almost equal to the collector current and the collector current was lower than that shown in Fig. 11(a). Although the alignment was conducted similarly to that for the $500-\mu$ m-pitch emitter chip, the diameter of the extractor grid is $200 \mu \mathrm{m}$, which is $100 \mu \mathrm{m}$ smaller than that for the $500-\mu \mathrm{m}$ pitch emitter chip, resulting in the larger number of collisions with the extractor grid. Since the radii of the extractor grid hole are 100 and $150 \mu \mathrm{m}$ for the 250 - and $500-\mu \mathrm{m}$-pitch extractor grids, respectively, the thickness of the extractor is $80 \mu \mathrm{m}$ for both the extractor grids, and the gap distance is $200 \mu \mathrm{m}$; the divergence angle of ion beams is estimated to be more than $20 \mathrm{deg}$. However, most of the beams are within the divergence angle of $30 \mathrm{deg}$. Since the pitch is 250 $\mu \mathrm{m}$, it is almost impossible to increase the diameter of each hole of the extractor grid to more than $200 \mu \mathrm{m}$. This result indicates that at least $300 \mu \mathrm{m}$ diameter is desirable for the extractor grid hole and thus the minimum pitch of emitters is estimated to be about $400 \mu \mathrm{m}$, considering an extra margin due to the ion beam divergence.

Moreover, we also tried to extract ion beams from the emitter chip fabricated without the Bosch process, as shown in Fig. 14. However, short circuits very frequently occurred and 
we were unable to obtain stable voltage-current characteristics. Since the height of the emitter tip was only $70 \mu \mathrm{m}$, the ionic liquid was probably extracted not only from the emitter tip but also from the reservoir directly to the extractor grid, as similarly observed in the preliminary experiment. This result indicates that the Bosch process is indispensable for the ionic liquid reservoir, which prevents abnormal ion extraction from the reservoir to the extractor grid.

4.3.2 Frequency dependence. Figure 15 shows the collector currents of cations and anions as functions bipolar pulse frequency at $V_{\text {ap }}=2000 \mathrm{~V}$, at which a stable current was obtained. Note that current was measured with a digital multimeter between 0.1 and $2 \mathrm{~Hz}$, but with an oscilloscope (Tektronix DPO4104B) at $50 \mathrm{~Hz}$ using a $100 \mathrm{k} \Omega$ shunt resistor, owing to the low sampling rate of the multimeter $(>30 \mathrm{~ms})$. Current seemed to be almost independent of bipolar pulse frequency, and ion emission was stable at lower frequencies. For ionic liquid ion sources, double layers occur at the interface between the electrode and the liquid. Since the width of the double layer is very small, even a small potential difference due to charge accumulation results in a strong electric field, which can cause undesired reactions, i.e., corrosion of the electrode and ionic liquid. This double-layer charging time is on the order of $10 \mathrm{~s}$; approximately $1 \mathrm{~Hz}$ alternating frequency can prevent the degradation of ion emission. ${ }^{37)}$ As shown in Fig. 15, the frequency examined in this study is within the range for stable operation, and this tendency is consistent with the report in the literature.

However, both the positive and negative currents at $50 \mathrm{~Hz}$ are slightly lower than those at lower frequencies. The reason is indicated in Fig. 16, which shows the applied voltage, 
collector current, and extractor current as functions of time. The data were obtained with an oscilloscope and averaged over 128 repetitions. We see the characteristics of the capacitor and inductor of the measurement circuit at the time of voltage alternation, where high-frequency harmonics are included. After the appearance of the circuit characteristics, we observed that the currents of the collector and extractor increased with time, indicating that the emission of the ionic liquid did not reach the steady state during this short period. At higher frequencies, we were only able to obtain transient emissions of ions, so that the current decreased at the same voltage as that at lower frequencies.

Finally, we also measured the voltage-current characteristics of applied DC voltage, which are shown in Fig. 17. Although this figure shows the current for only 60 s, we observed almost the same current for more than 10 min. However, the current was not stable compared with that for the measurement of voltage alternation, implying that the degradation of ion emission occurred owing to the double layer at the emitter tips. In terms of beam neutralization, a higher frequency is desirable for preventing inequalities in beam current if a single unit of a thruster is used in space. However, it would result in a lower beam current. If two thrusters are operated at lower frequencies, it would be useful to simultaneously extract cations from one of the thrusters and anions from the other thruster to maintain current balance and charge neutrality.

\section{Summary}

To develop ILESTs that can be mounted on miniature satellites lighter than about $10 \mathrm{~kg}$, we 
have fabricated the emitter chip and extractor grid, and measured the ion beam currents of the ILEST. In the preliminary experiment, we fabricated four emitters on a silicon wafer with an 8-mm-diameter reservoir, and we confirmed that ions were successfully extracted from the ILEST, but half of the ion beams collided against the extractor grid. The thrust estimated was less than $1 \mu \mathrm{N}$, which was too low even for nanosatellites. To increase the thrust and to decrease the extractor current, we fabricated a dense emitter array and improved the alignment system between the emitter and the extractor grid. We have measured the dependences of voltagecurrent characteristics on the emitter-extractor gap distance and the frequency of bipolar pulse voltage. Beam current did not increase with increasing number of emitters, probably owing to the lack in uniformity of the emitter tips. The increase in gap distance resulted in the increase in the starting voltage of ion extraction and collector currents. There was a stable region of applied voltages higher than the starting voltage for ion beam extraction; beam currents became unstable at voltages higher than the stable region. The beam currents were almost independent of bipolar pulse frequency. However, at frequencies higher than about $10 \mathrm{~Hz}$, ion current did not reach the steady state and was lower than those at lower frequencies, implying that frequencies lower than about $10 \mathrm{~Hz}$ are desirable for extracting ion beams for voltage alternation. For emitter configuration, the reservoir layer was found to be indispensable for preventing undesirable short circuits between the extractor and the emitter. Moreover, because of the beam divergence, the minimum emitter pitch to prevent significant beam collisions against the extractor grid was estimated to be about $400 \mu \mathrm{m}$. 
It is also important to measure other ion beam characteristics, such as beam divergence angle and extracted ion energy. Moreover, extracted and emitted ions are composed of not only monomers but also dimers, trimers, or droplets under certain conditions for electrospray thrusters using ionic liquids. Since the mass-to-charge ratio of ions is related to the thruster parameter in Eqs. (1) and (2), ToF measurement is required for investigating the mass of extracted ions and estimate the thruster performance. These are left for future work, results of which will be published elsewhere.

\section{Acknowledgments}

This work was financially supported in part by JSPS KAKENHI Grant Number JP15K14247 and the Asahi Glass Foundation. This work was also supported by Kyoto University Nano Technology Hub in "Nanotechnology Platform Project" sponsored by the Ministry of Education, Culture, Sports, Science and Technology, Japan (MEXT), and 4-University Nano/Micro Fabrication Consortium.

\section{References}

1) T. Suzuki, M. Hara, H. Oguchi, and H. Kuwano, Sens. Actuators A 215, 161 (2014).

2) M. Takeuchi, Y. Hoshide, H. Ryuto, and G. H. Takaoka, J. Vac. Sci. Technol. A 34, 02 D108 (2016).

3) M. Takeuchi, Y. Hoshide, T. Hamaguchi, H. Ryuto, and G. H. Takaoka, Trans. Mater. Res. Soc. Jpn. 40, 87 (2015).

4) J. B. Fenn, M. Mann, C. K. Meng, S. F. Wong, and C. M. Whitehouse, Science 246, 64 (1989).

5) K. Tanaka, H. Waki, Y. Ido, S. Akita, Y. Yoshida, T. Yoshida, and T. Matsuo, Rapid Commun. Mass Spectrom. 2, 151 (1988). 
6) M. Tajmar, A. Genovese, and W. Steiger, J. Propul. Power 20, 211 (2004).

7) Y. Takao, K. Eriguchi, and K. Ono, J. Appl. Phys. 101, 123307 (2007).

8) M. Auweter-Kurtz, B. Glocker, T. Goelz, H. L. Kurtz, E. W. Messerschmid, M. Riehle, and D. M. Zube, J. Propul. Power 12, 1077 (1996).

9) W. E. Morren, T. W. Haag, J. S. Sovey, and S. S. Hay, J. Propul. Power 5, 197 (1989).

10) H. Koizumi and H. Kuninaka, J. Propul. Power 26, 601 (2010).

11) A. Smirnov, Y. Raitses, and N. J. Fisch, J. Appl. Phys. 95, 2283 (2004).

12) A. Sasoh, Phys. Plasmas 1, 464 (1994).

13) K. Takahashi, T. Lafleur, C. Charles, P. Alexander, R. W. Boswell, M. Perren, R. Laine, S. Pottinger, V. Lappas, T. Harle, and D. Lamprou, Appl. Phys. Lett. 98, 141503 (2011).

14) D. M. Goebel and I. Katz, Fundamentals of Electric Propulsion: Ion and Hall Thrusters (Wiley, Hoboken, NJ, 2008).

15) W. P. Wright and P. Ferrer, Prog. Aerosp. Sci. 74, 48 (2015).

16) H. Kuninaka, K. Nishiyama, I. Funaki, T. Yamada, Y. Shimizu, and J. Kawaguchi, J. Propul. Power 23, 544 (2007).

17) H. Koizumi, H. Kawahara, K. Yaginuma, J. Asakawa, Y. Nakagawa, Y. Nakamura, S. Kojima, T. Matsuguma, R. Funase, J. Nakatsuka, and K. Komurasaki, Trans. Jpn. Soc. Aeronaut. Space Sci., Aerosp. Technol. Jpn. 14, Pb_13 (2016).

18) T. Toizumi, T. Enomoto, Y. Yatsu, T. Nakamori, N. Kawai, K. Ishizaka, A. Muta, H. Morishita, K. Akiyama, N. Kisa, S. Inagawa, M. Kawakubo, J. Nishida, S. Mizunuma, S. Matsunaga, and J. Kataoka, Physica E 43, 685 (2011).

19) S.-I. Nishida, S. Kawamoto, Y. Okawa, F. Terui, and S. Kitamura, Acta Astronaut. 65, 95 (2009).

20) S. Rucinski, K. Carroll, R. Kuschnig, J. Matthews, and P. Stibrany, Adv. Space Res. 31, 371 (2003).

21) H. Koizumi, K. Komurasaki, J. Aoyama, and K. Yamaguchi, Trans. Jpn. Soc. Aeronaut. Space Sci., Aerosp. Technol. Jpn. 12, Tb_19(2014).

22) M. M. Micci and A. D. Ketsdever, Micropropulsion for Small Spacecraft (American Institute of Aeronautics and Astronautics, Reston, VA, 2000). 
23) M. Watanabe, Hyomen Kagaku 28, 298 (2007) [in Japanese].

24) D. R. MacFarlane, N. Tachikawa, M. Forsyth, J. M. Pringle, P. C. Howlett, G. D. Elliott, J. H. Davis, M. Watanabe, P. Simon, and C. A. Angell, Energy Environ. Sci. 7, 232 (2013).

25) M. Armand, F. Endres, D. R. MacFarlane, H. Ohno, and B. Scrosati, Nat. Mater. 8, 621 (2009).

26) M. Yamagata, K. Tanaka, Y. Tsuruda, Y. Sone, S. Fukuda, S. Nakasuka, M. Kono, and M. Ishikawa, Electrochemistry 83, 918 (2015).

27) S. Imaizumi, Y. Ohtsuki, T. Yasuda, H. Kokubo, and M. Watanabe, ACS Appl. Mater. Interfaces 5, 6307 (2013).

28) R. Kawano, H. Matsui, C. Matsuyama, A. Sato, M. A. B. H. Susan, N. Tanabe, and M. Watanabe, J. Photochem. Photobiol. C 164, 87 (2004).

29) G. Taylor, Proc. R. Soc. London A 280, 383 (1964).

30) R. S. Legge and P. C. Lozano, J. Propul. Power 27, 485 (2011).

31) S. Dandavino, C. Ataman, S. Chakraborty, H. Shea, C. Ryan, and J. Stark, 33rd International Electric Propulsion Conference, 2013, IEPC.

32) P. Lozano, M. Martínez-Sánchez, and J. M. Lopez-Urdiales, J. Colloid Interface Sci. 276, 392 (2004).

33) C. Guerra-Garcia, D. Krejci, and P. Lozano, J. Phys. D 49, 115503 (2016).

34) S. Dandavino, C. Ataman, C. N. Ryan, S. Chakraborty, D. Courtney, J. P. W. Stark, and H. Shea, J. Micromech. Microeng. 24, 075011 (2014).

35) D. G. Courtney and P. Lozano, Trans. Jpn. Soc. Aeronaut. Space Sci., Aerosp. Technol. Jpn. 8, Pb_73 (2010).

36) C. S. Perez-Martinez and P. C. Lozano, J. Vac. Sci. Technol. B 30, 06 F601 (2012).

37) P. Lozano and M. Martínez-Sánchez, J. Colloid Interface Sci. 280, 149 (2004). 


\section{List of Figure Captions}

Fig. 1. (Color online) Schematic of the ILEST. High positive/negative voltage extracts positive/negative ions (cations/anions) from the ionic liquid.

Fig. 2. Structural formula of EMI-BF4.

Fig. 3. (Color online) Fabrication procedure for an emitter chip. (a) Firstly, we prepare a clean silicon wafer. (b) A thick positive-type photoresist (PMER P-LA900PM) is applied to the wafer and heated, and (c) photolithographed using a patterned photomask. (d) Isotropic etching using $\mathrm{SF}_{6}$ to form the cone shape of the emitter tips. (e) Using the Bosch process, the height of emitters is increased and a reservoir is fabricated. (f) Finally, the thick photoresist layer is removed.

Fig. 4. (Color online) (a) Photograph of the emitter chip $\left(1 \times 1 \mathrm{~cm}^{2}\right)$, (b) SEM image of the emitters fabricated on silicon substrate $(\times 150)$, and (c) enlarged SEM view of one of the emitters $(\times 700)$.

Fig. 5. (Color online) (a) Schematic of the experimental setup for the current measurements and the configuration of each electrode; (b) the extractor grid using a TEM grid. 
Fig. 6. (Color online) Collector current (solid circles) and extractor current (open triangles) as functions of applied voltage.

Fig. 7. Mask layouts of 250- and 500- $\mu$ m-pitch emitter chips. The diameters of the open, solid gray, and solid black circles are 100, 110, and $120 \mu \mathrm{m}$, respectively.

Fig. 8. SEM images of the emitter array with $250 \mu \mathrm{m}$ pitch $(\times 150)$ in (a) the outermost, (b) the middle, and (c) the center area inside the reservoir.

Fig. 9. (Color online) Photograph of the emitter chip fabricated on a silicon wafer and the extractor grid made of stainless steel with a thickness of $80 \mu \mathrm{m}$. Both chips are $1 \mathrm{~cm}$ by $3 \mathrm{~cm}$.

Fig. 10. (Color online) (a) Cross-sectional view of the alignment system of the emitter chip and the extractor grid and (b) the wire diagram for the current measurement.

Fig. 11. (Color online) Collector current (solid circles) and extractor current (open triangles) as functions of applied voltage for the $500-\mu \mathrm{m}$-pitch emitter chip. The gap distances between the collector and extractor, $d$, are (a) 0.2 , (b) 0.3 , and (c) $0.4 \mathrm{~mm}$.

Fig. 12. (Color online) Applied voltage (dash-dotted line), collector current (solid line), and 
extractor current (dotted line) as functions of time at (a) $V_{\text {ap }}=2200 \mathrm{~V}$ and (b) $V_{\text {ap }}=3000 \mathrm{~V}$ for the 500- $\mu \mathrm{m}$-pitch emitter chip with $d=0.2 \mathrm{~mm}$.

Fig. 13. (Color online) Collector current (solid circles) and extractor current (open triangles) as functions of applied voltage for the $250-\mu \mathrm{m}$-pitch emitter chip with $d=0.2 \mathrm{~mm}$.

Fig. 14. (a) SEM images of the emitter array with $500 \mu \mathrm{m}$ pitch fabricated without the Bosch process $(\times 15)$ and $(b)$ enlarged view of one of the emitters $(\times 500)$.

Fig. 15. (Color online) Collector currents of cations (solid circles) and anions (open circles) as functions of bipolar pulse frequency at $V_{\text {ap }}=2000 \mathrm{~V}$ for the $500-\mu \mathrm{m}$-pitch emitter chip with $d$ $=0.2 \mathrm{~mm}$.

Fig. 16. (Color online) Applied voltage (dash-dotted line), collector current (solid line), and extractor current (dashed line) as functions of time at a bipolar pulse frequency of $50 \mathrm{~Hz}$ in Fig. 15.

Fig. 17. (Color online) Applied voltage (dash-dotted line), collector current (solid line), and extractor current (dashed line) as functions of time for the 500- $\mu \mathrm{m}$-pitch emitter chip with $d=$ $0.2 \mathrm{~mm}$ when DC voltage of $2050 \mathrm{~V}$ is applied for more than $10 \mathrm{~min}$. 

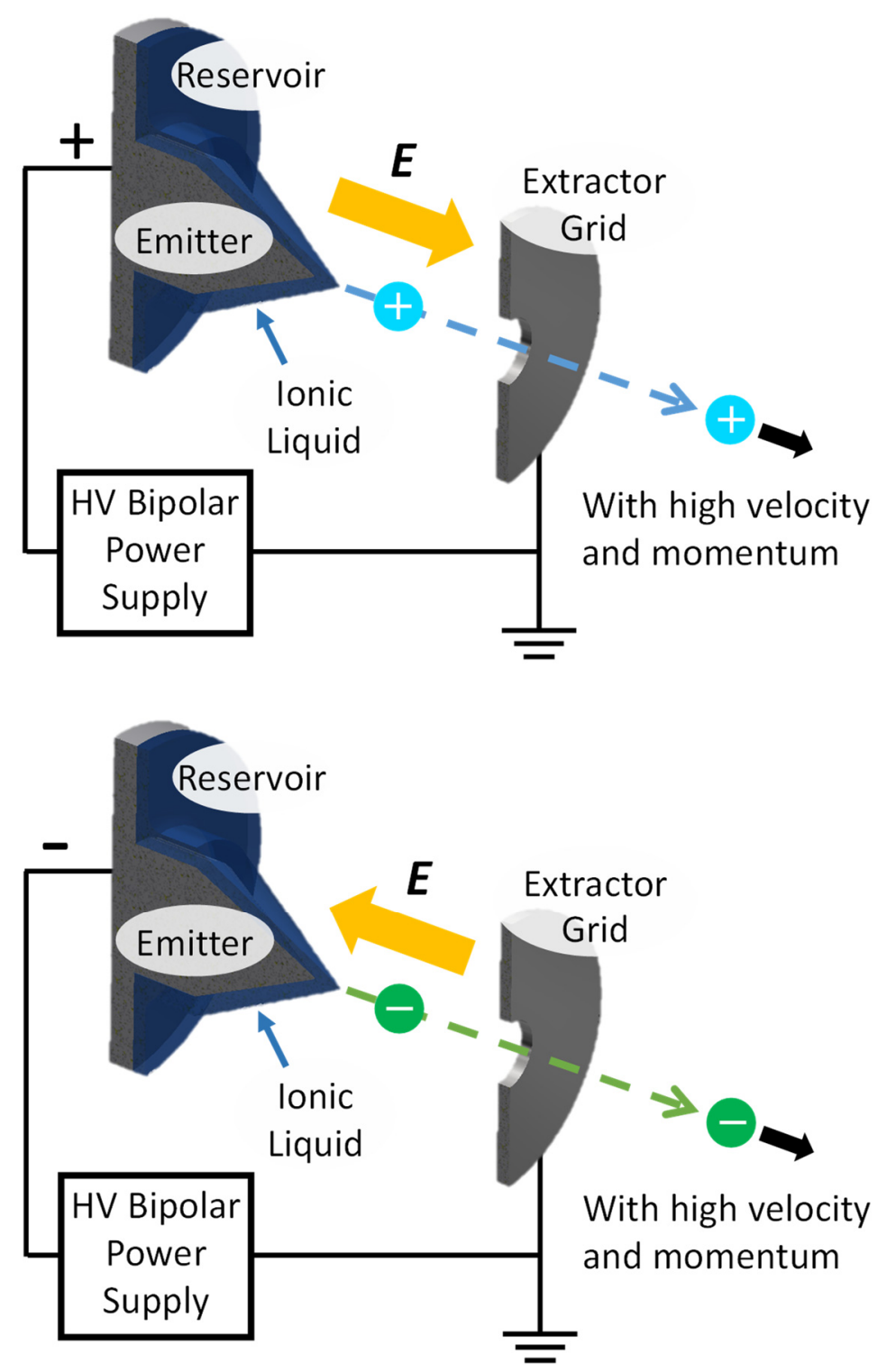

Fig. 1 
$\widehat{\mathrm{N}_{\mathrm{N}}^{+}} \widehat{\mathrm{N}}^{+}+\mathrm{BF}_{4}$

Fig. 2 
(a)

Si

Thick photoresist

(b)

$\downarrow$

(c)

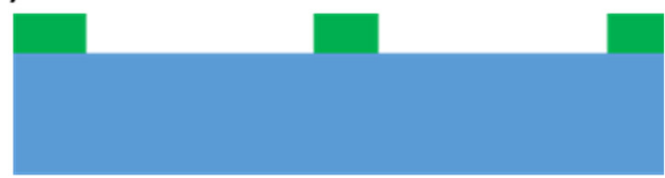

(d)

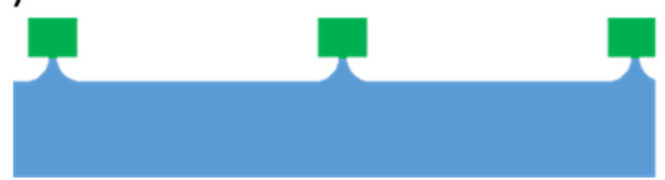

(e)

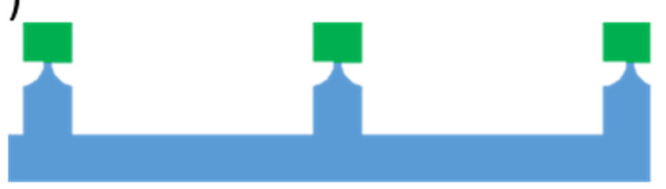

(f)

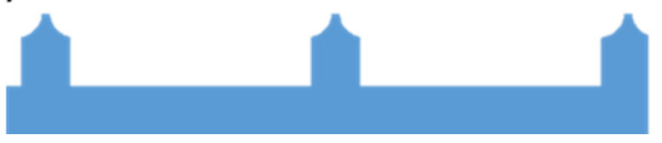

Fig. 3 

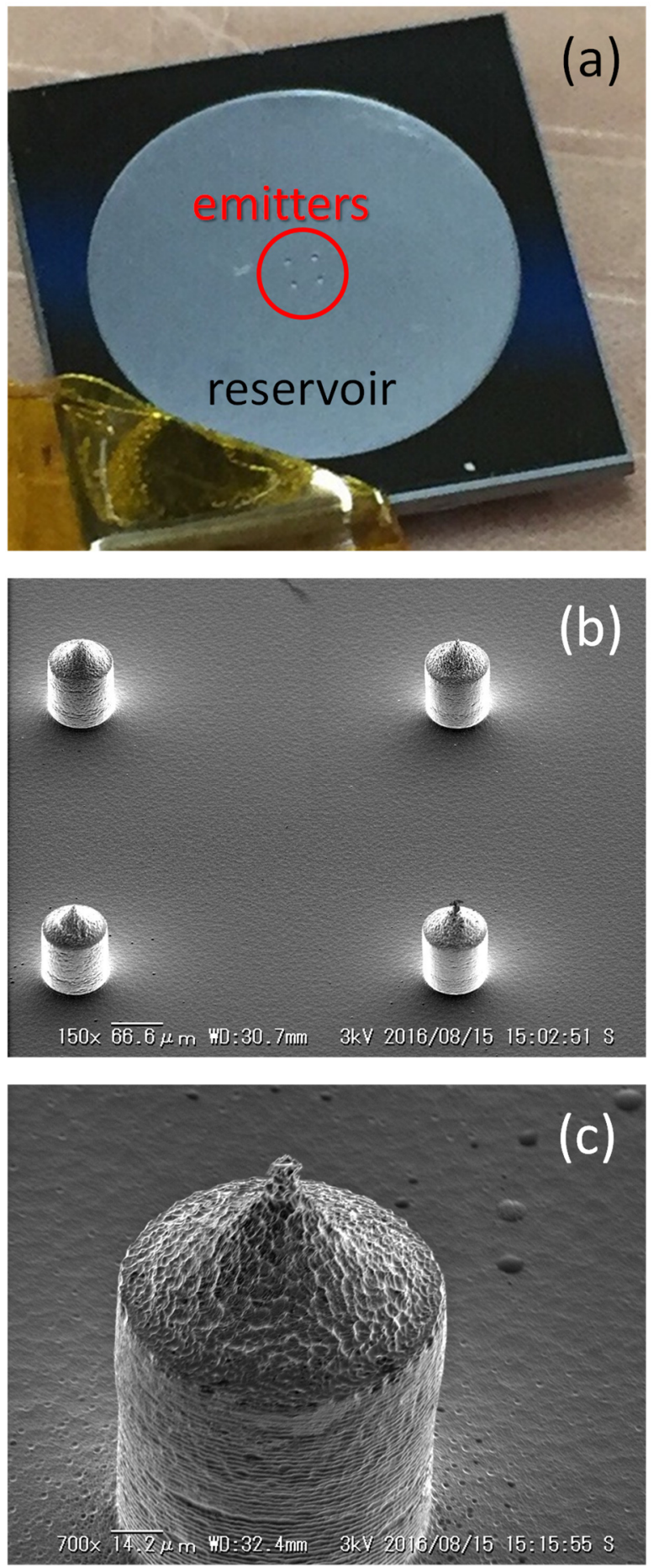

Fig. 4 


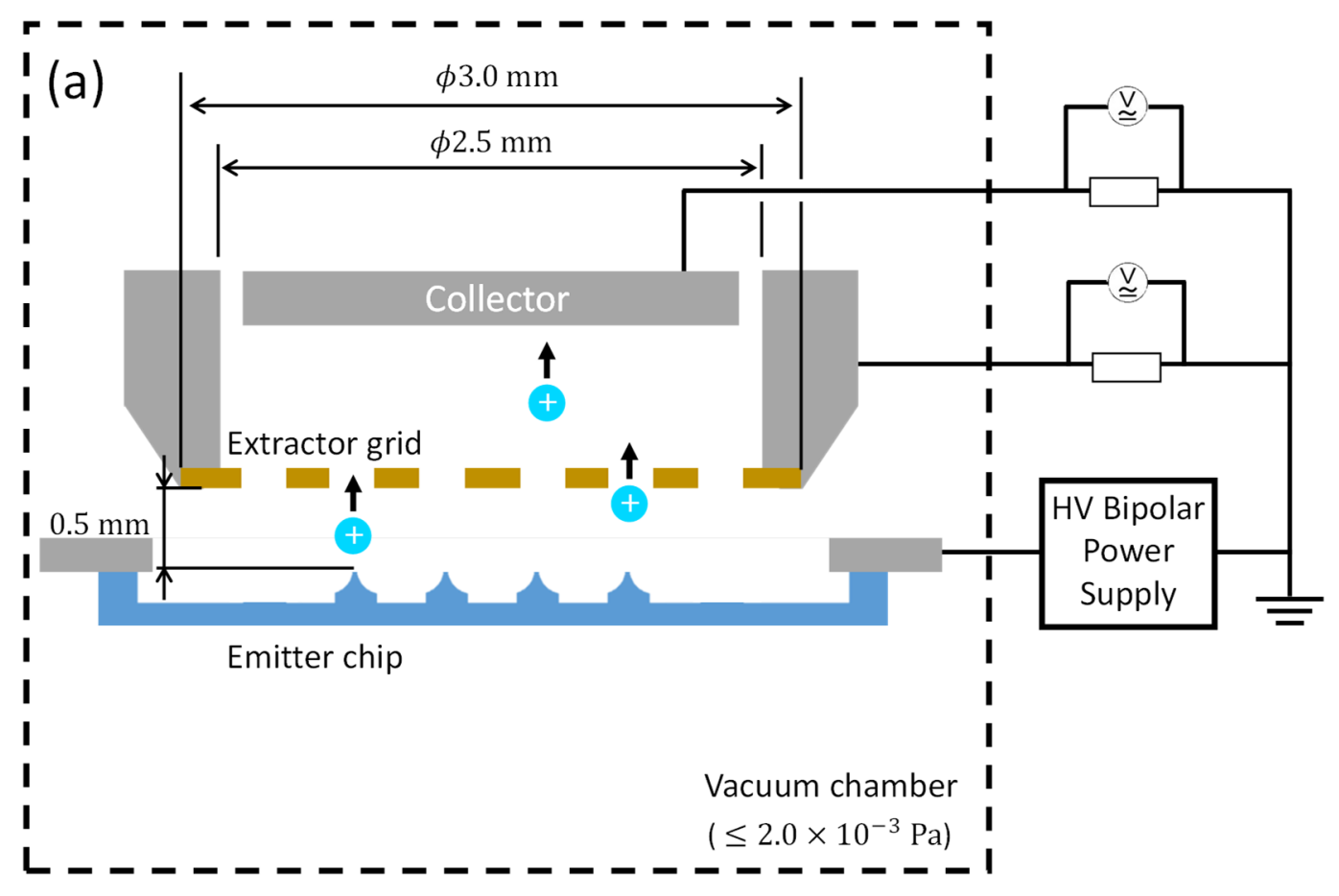

(b)

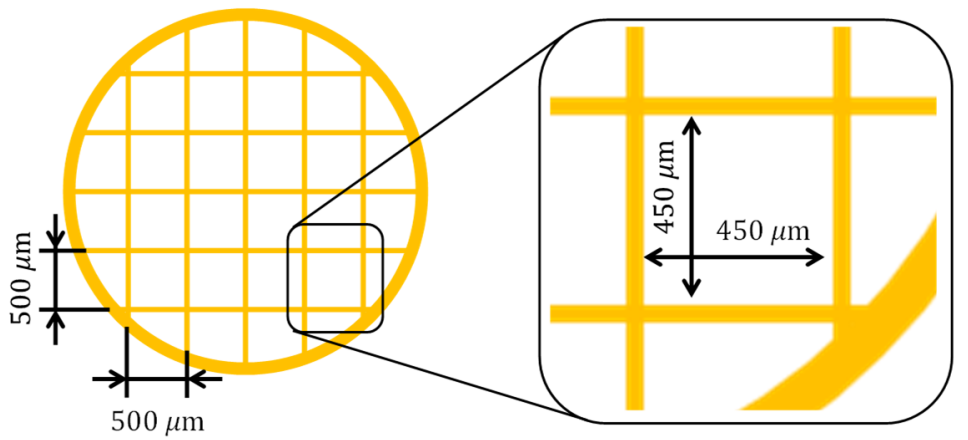

Fig. 5 


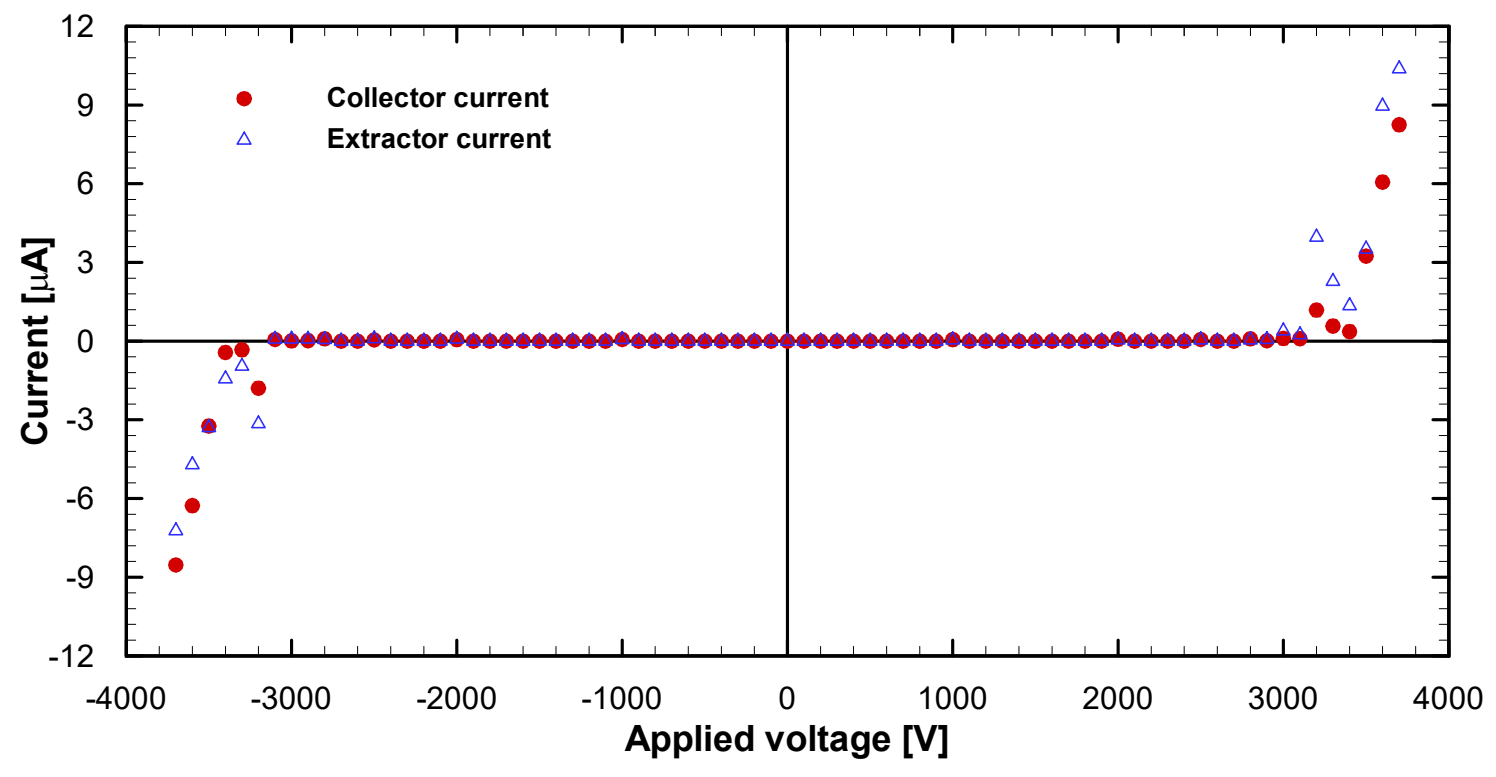

Fig. 6 


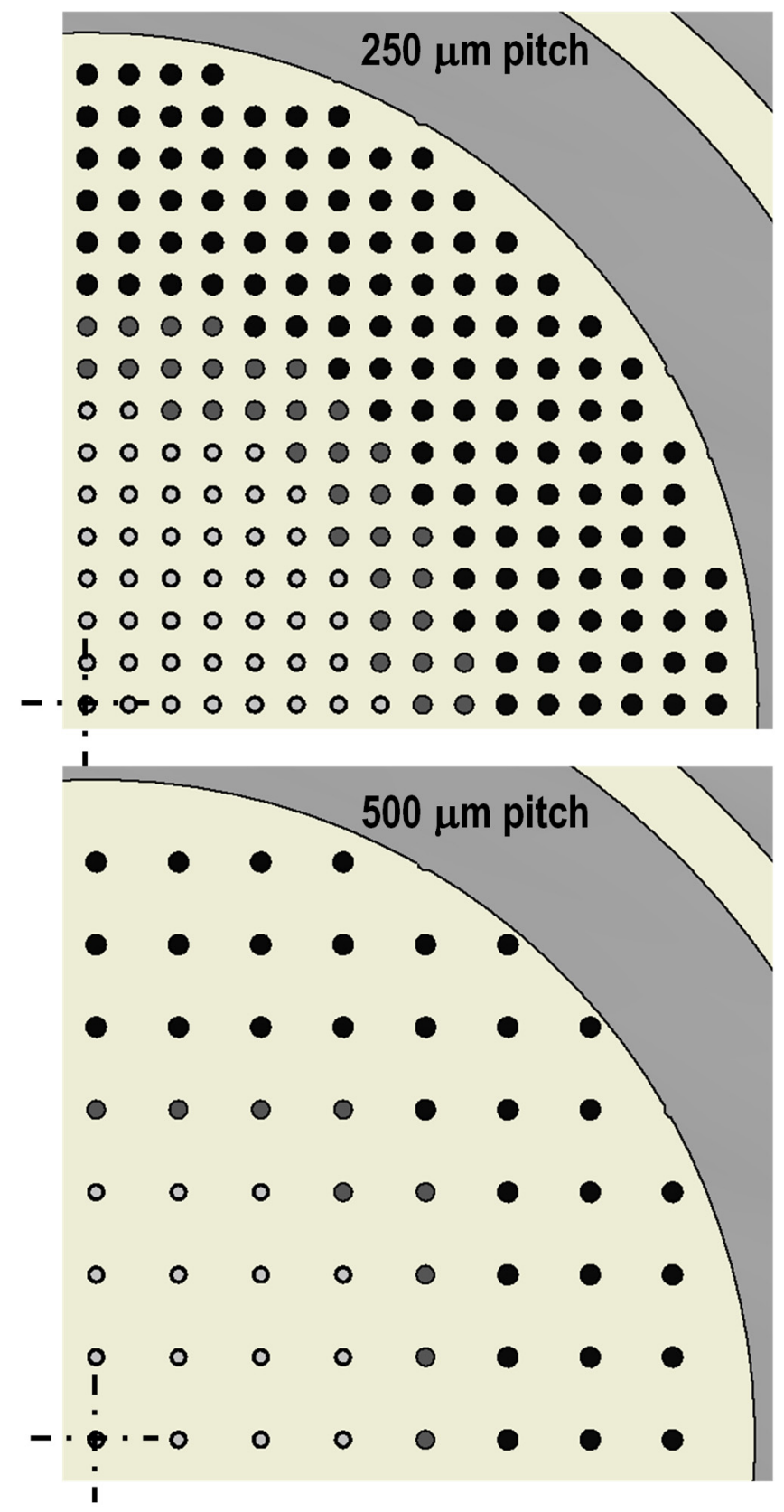

Fig. 7 

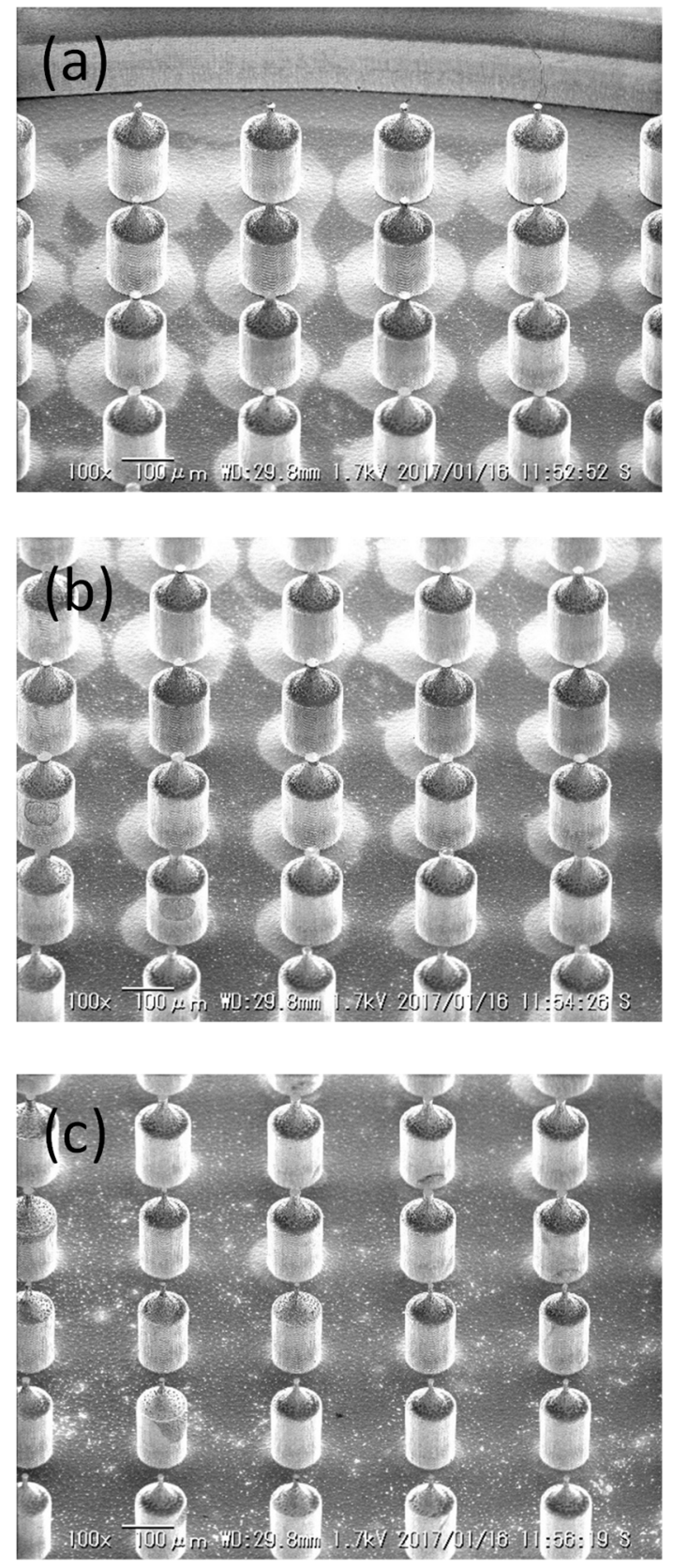

Fig. 8 


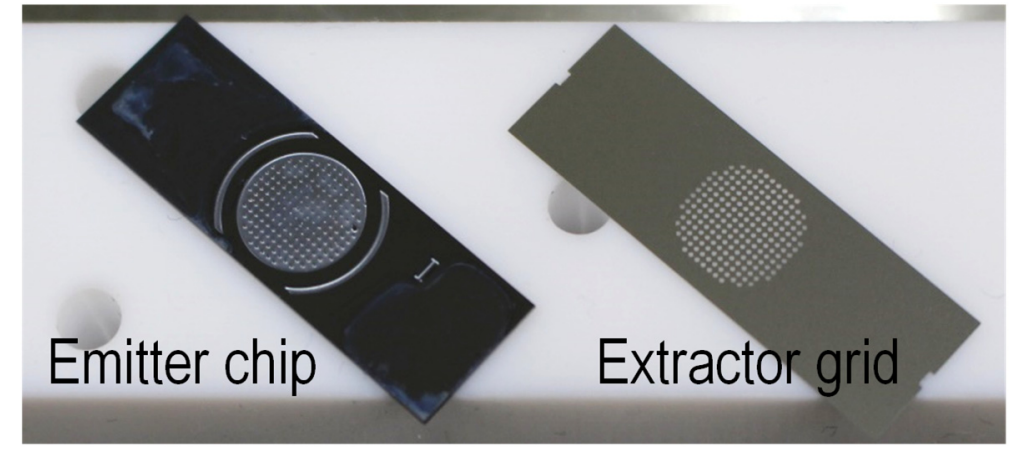

Fig. 9 
(a)

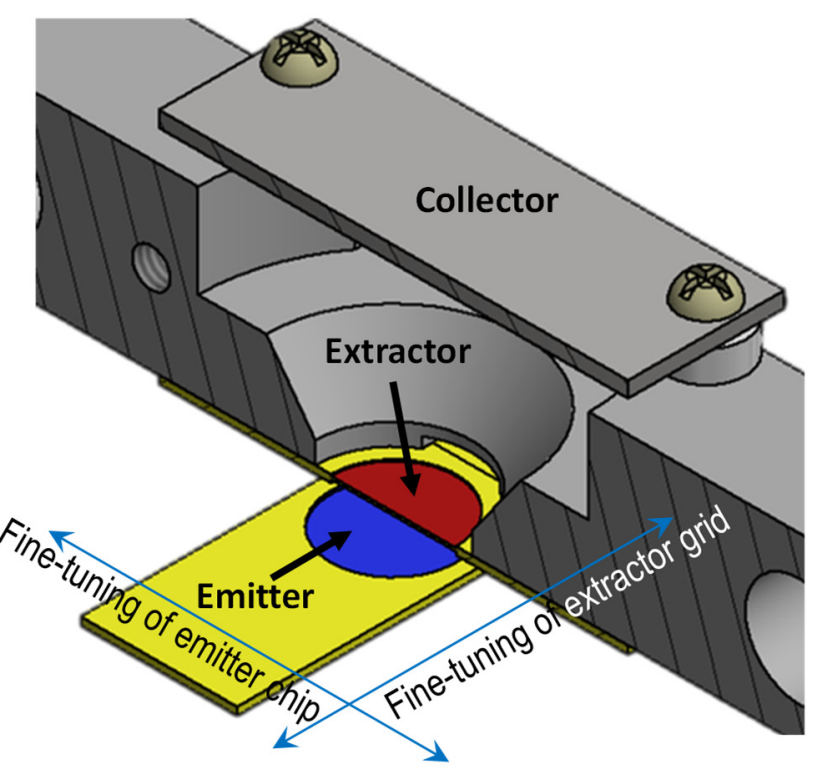

(b)

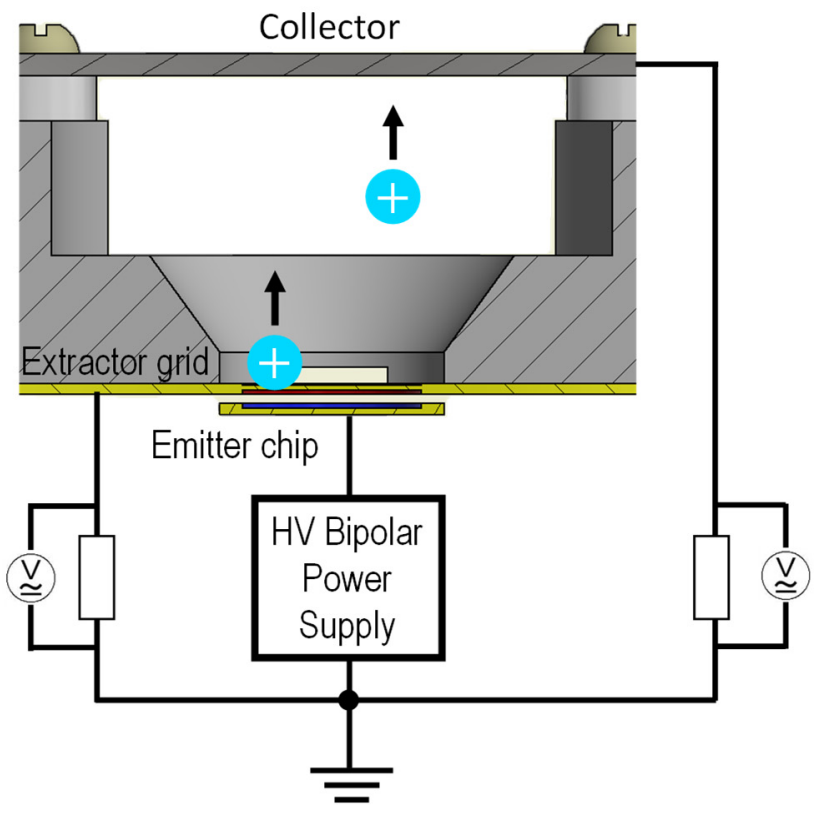

Fig. 10 

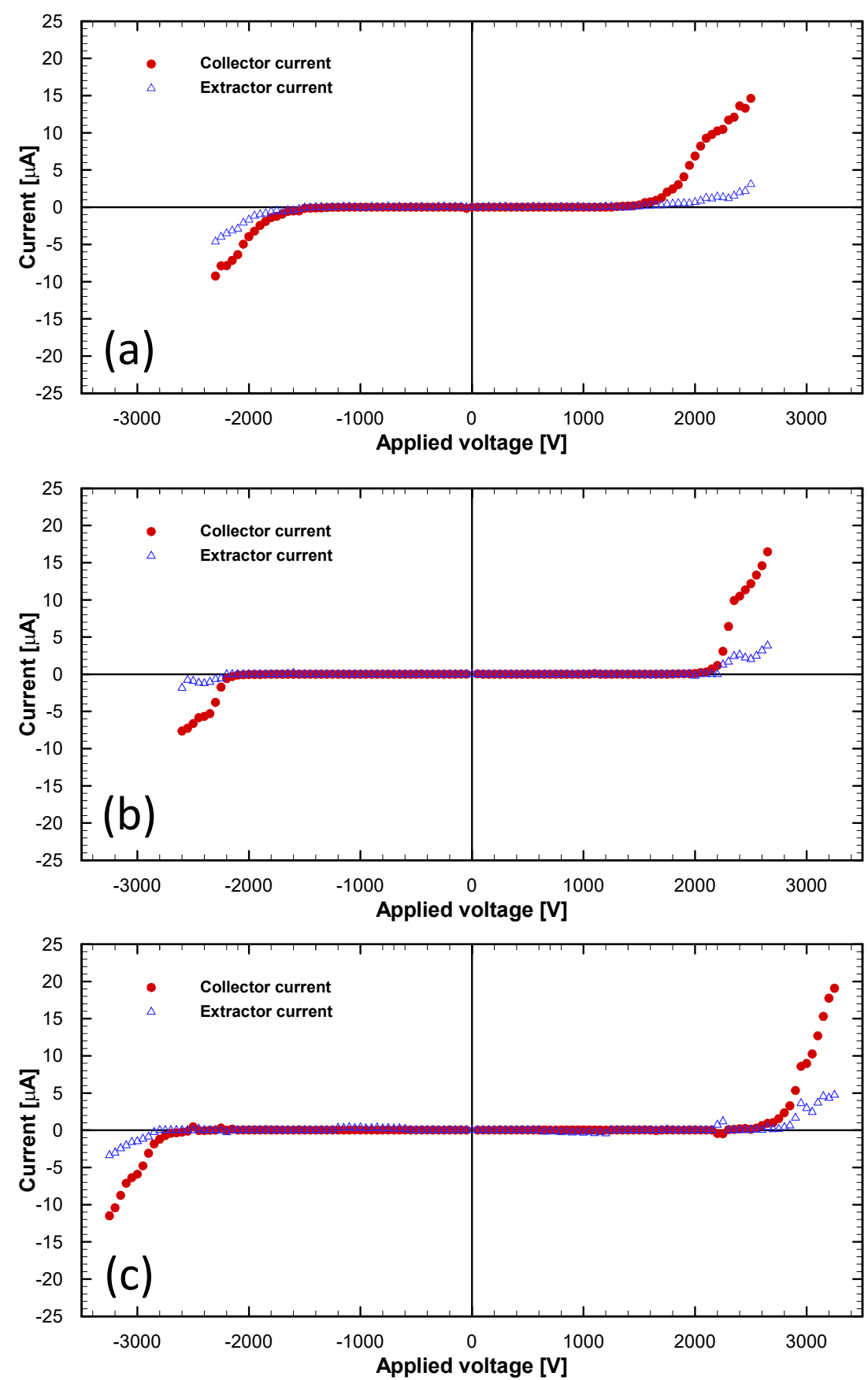

Fig. 11 

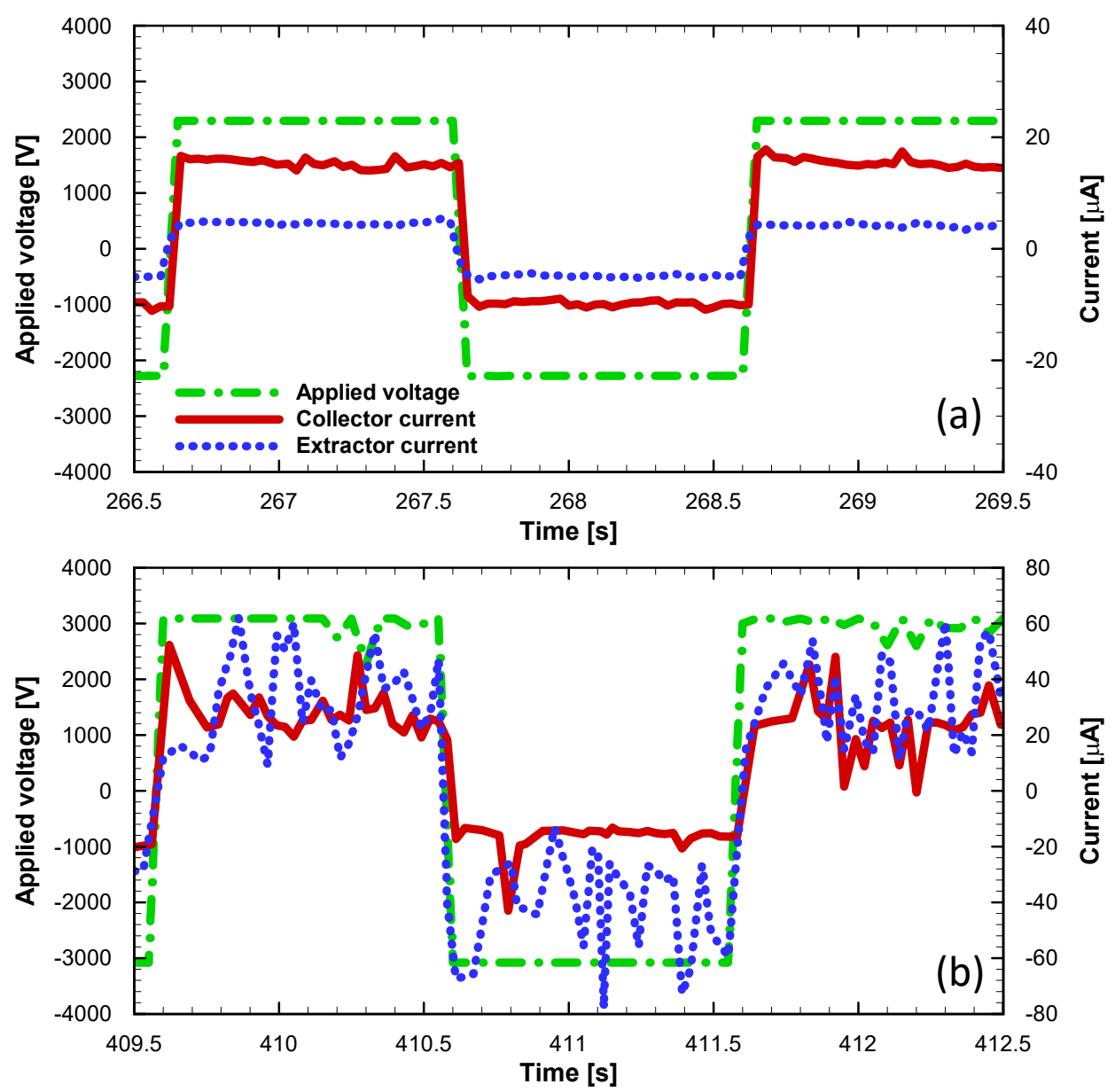

Fig. 12 


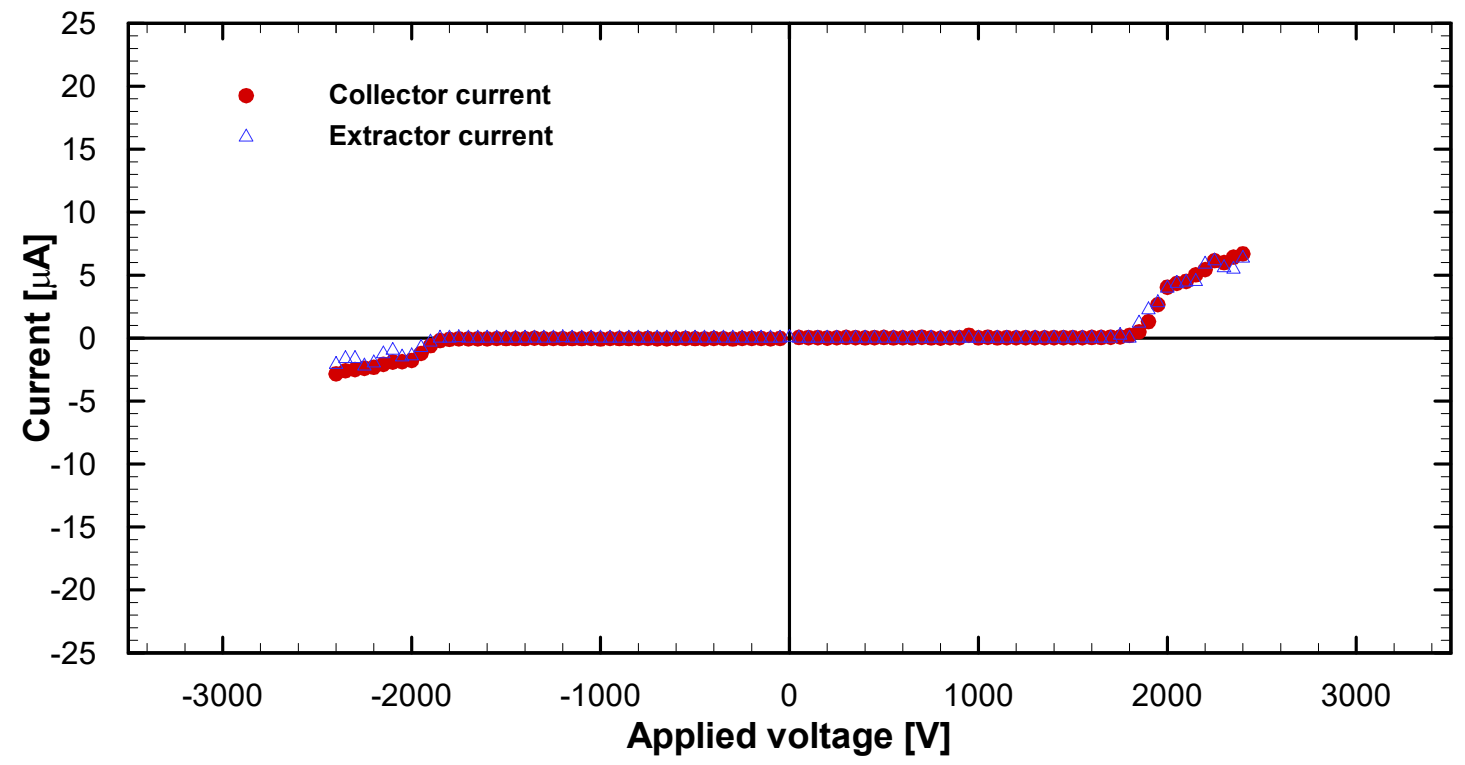

Fig. 13 

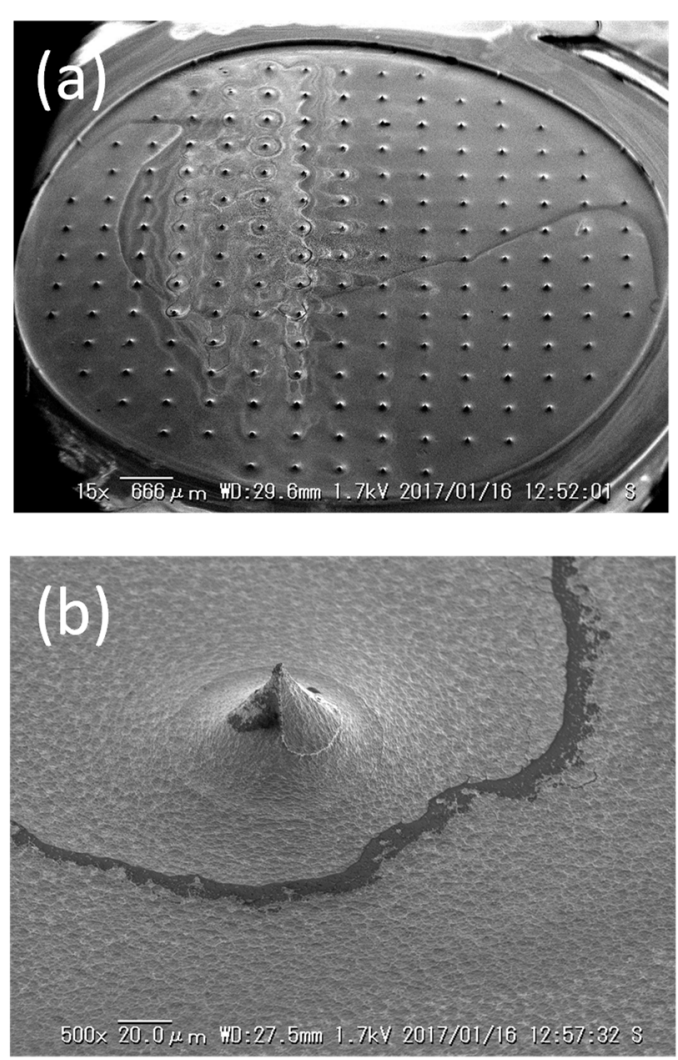

Fig. 14 


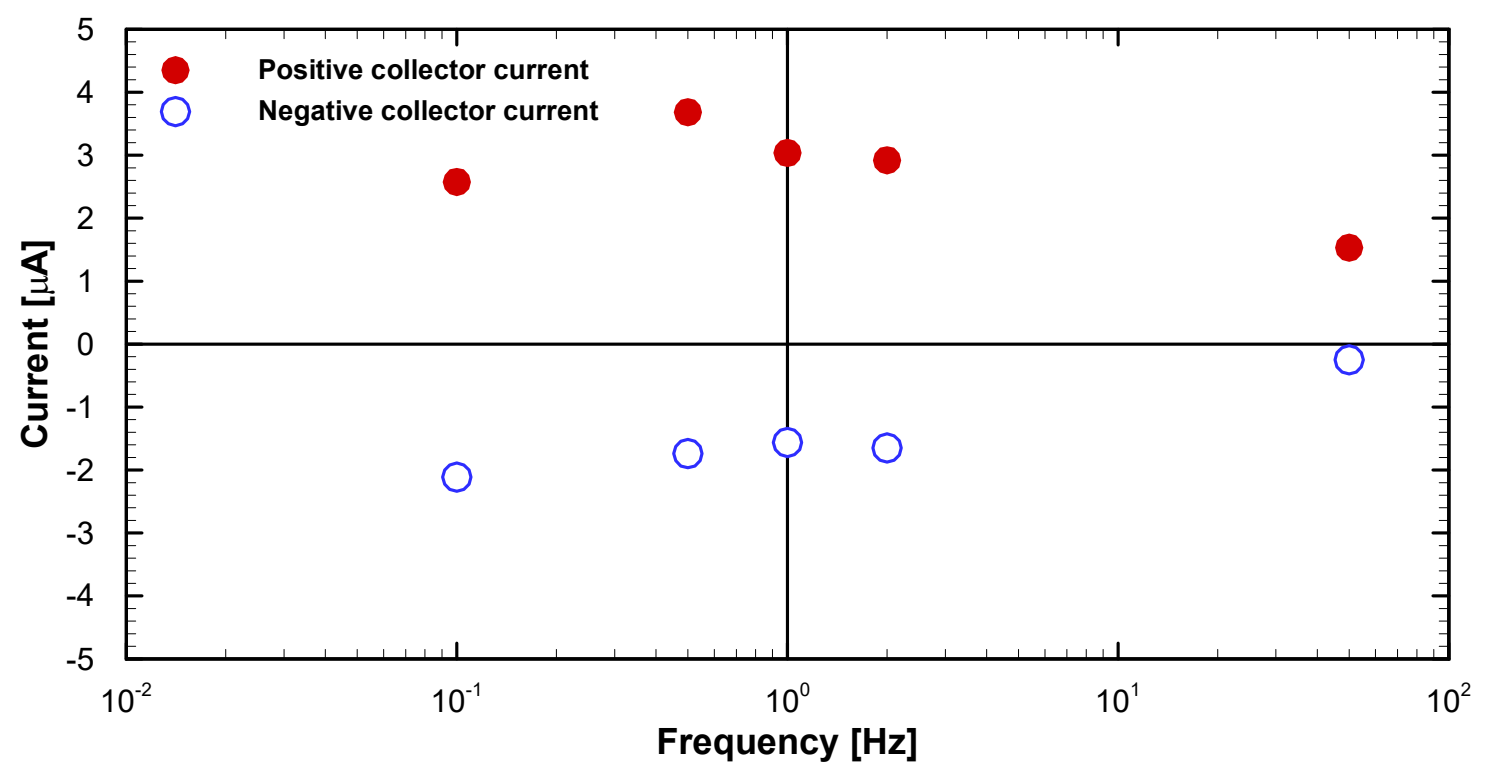

Fig. 15 


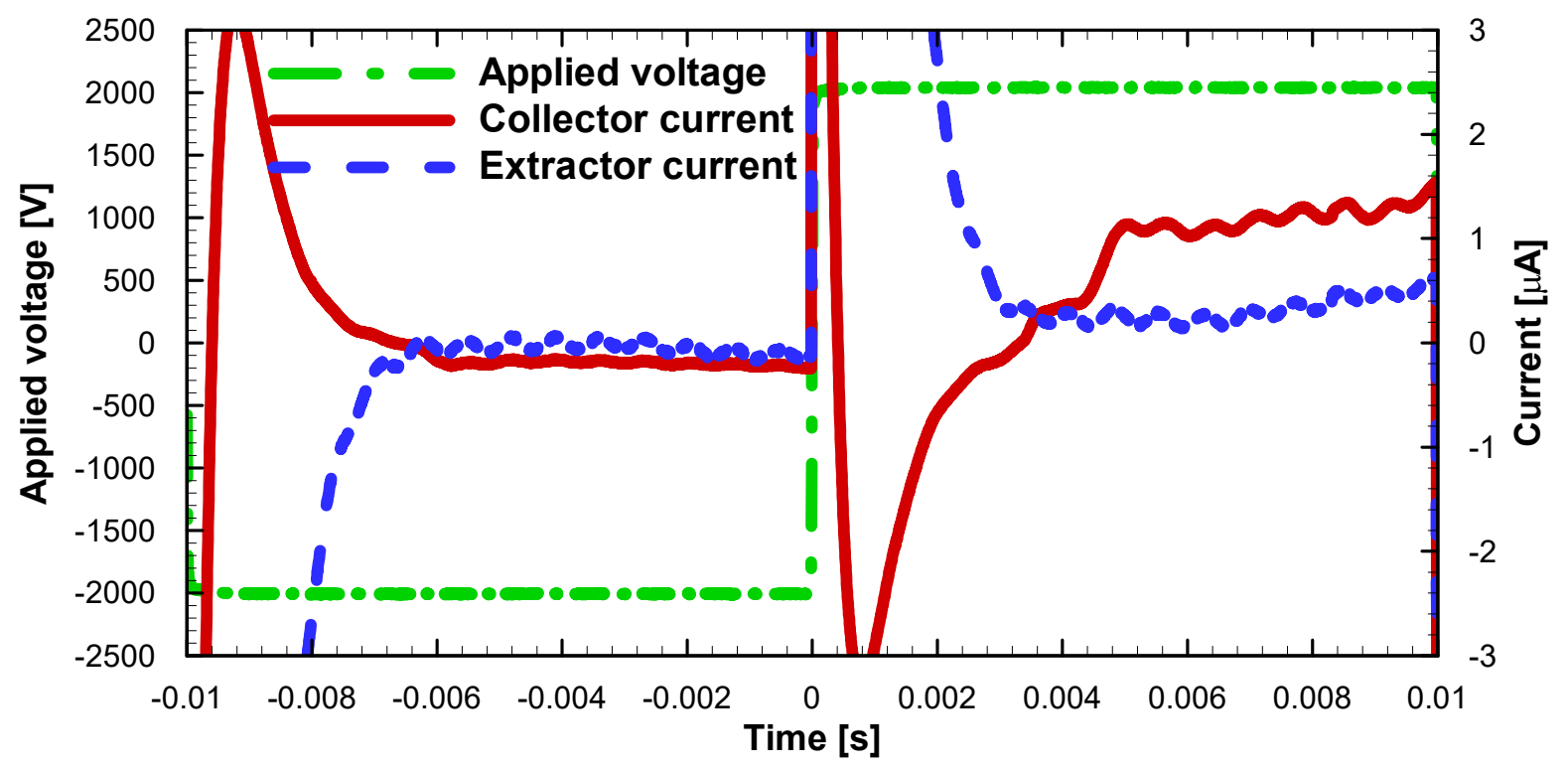

Fig. 16 


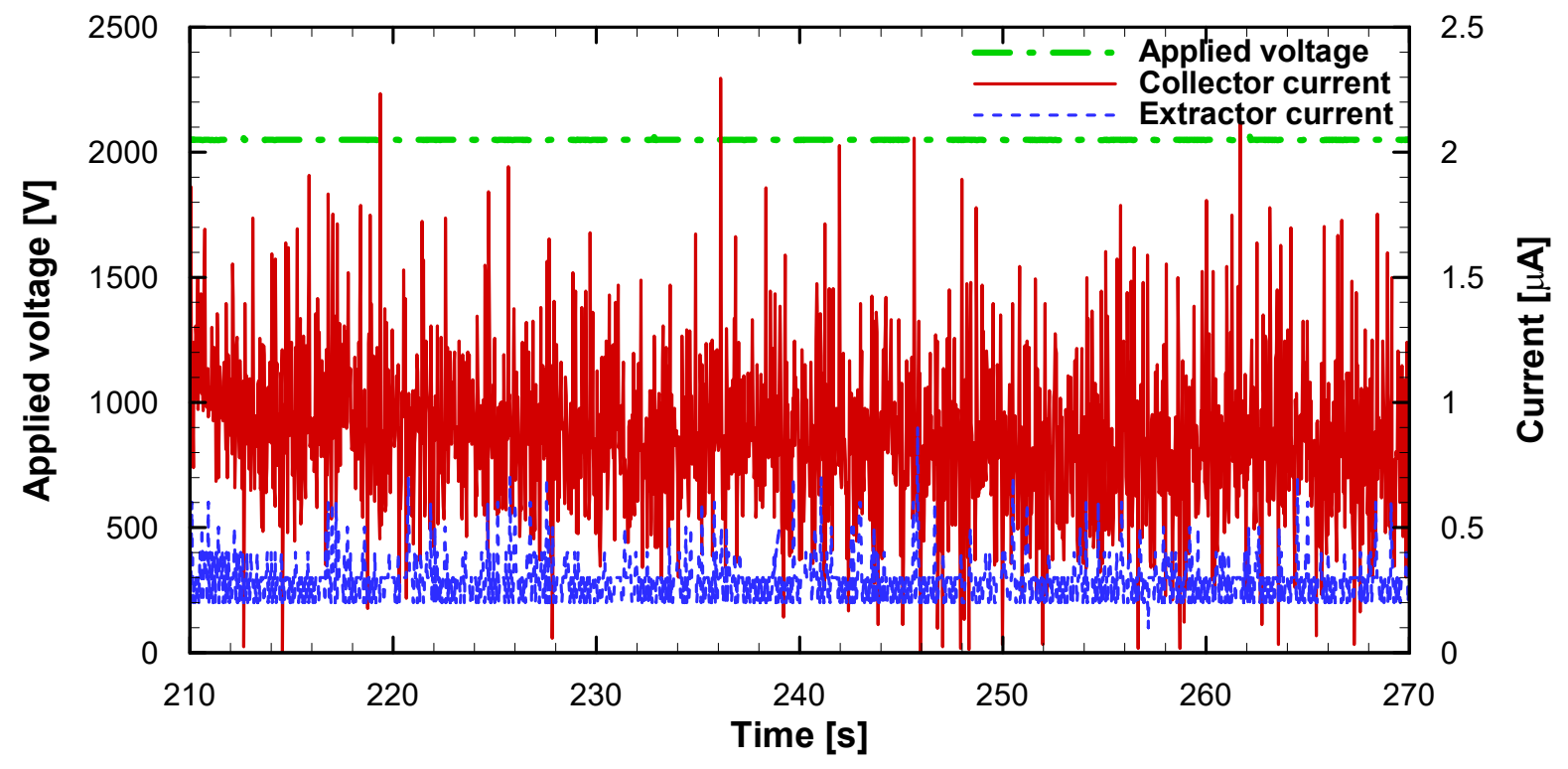

Fig. 17 